\title{
Logical and Algebraic Views of a Knot Fold of a Regular Heptagon
}

\author{
Fadoua Ghourabi ${ }^{1}$, Tetsuo Ida ${ }^{2}$ and Kazuko Takahashi ${ }^{1}$ \\ 1 Kwansei Gakuin University, Japan \\ ghourabi@kwansei.ac.jp, ktka@kwansei.ac.jp \\ 2 University of Tsukuba, Japan \\ ida@i-eos.org
}

\begin{abstract}
Making a knot on a rectangular origami or more generally on a tape of a finite length gives rise to a regular polygon. We present an automated algebraic proof that making two knots leads to a regular heptagon. Knot fold is regarded as a double fold operation coupled with Huzita's fold operations. We specify the construction by describing the geometrical constraints on the fold lines to be used for the construction of a knot. The algebraic interpretation of the logical formulas allows us to solve the problem of how to find the fold operations, i.e. to find concrete fold lines. The logical and algebraic framework incorporated in a system called Eos (e-origami system) is used to simulate the knot construction as well as to prove the correctness of the construction based on algebraic proof methods.
\end{abstract}

\section{Introduction}

From the early history of mathematics, the correspondence between geometry and algebra has been recognized and has been the subject of constant study. In some sense, ancient geometry was a mother of algebra. In particular, solving algebraic equations has been related to the realm of Euclidean geometry. Early mathematicians, notably Khawarizmi and Khayyam, gave geometrical meanings to the solutions of equations. Khayyam's insight was that solutions of certain cubic equations are points of intersections of conic sections [1. Seven centuries later, after freeing algebra from geometrical thinking, Wantzel proved that solving cubic solutions is impossible by Euclidean tools, i.e. compass and straightedge [2]. Further tools have been invented and used to perform constructions that are impossible by Euclidean tools.

Paper folding, i.e. origami, allows solving cubic equations and, hence, geometrical constructions such as trisection of an arbitrary angle or a regular heptagon are realizable. Given an origami paper, what we can do by hand is to construct creases and points. The creases are constructed by folding the paper along the lines which we call fold lines. The points are constructed by the intersection of lines. Huzita presented a set of fold operations (known also as Huzita's axioms in literature) with which he showed how to obtain fold lines [3. Huzita's fold operations are simple to perform by hand but powerful enough to solve cubic equations 4 .

The geometrical construction of a regular heptagon has a unique history in that it belongs to the famous classical impossible problems by Euclidean tools. Using paper folding, the construction was shown to be possible. In this paper, we show another method of constructing a regular heptagon based on an extension of Huzita's fold operations by introducing knotting. The constructions of regular $n$-gons (in particular of regular pentagon and heptagon) by making knots have not been rigorously specified, and the observation that their constructions purport to problems of solving geometrical constraints is missing. 
We investigate the geometrical constraints of the knot fold towards constructions of regular polygons and proofs of the correctness of these constructions. The knot fold operation is specified by an existentially quantified formula of the first-order predicate logic. The formula is then translated into a set of algebraic equalities and disequalities, which then are solved by specialized solvers based on Gröbner basis theory and/or CAD (Cylindrical Algebraic Decomposition). We have developed a computational origami system called Eos [5] using computer algebra system Mathematica. In brief, it is an e-origami system which allows us to do virtual origami. It has capabilities of constructing and visualizing origami geometrical objects, algebraically analyzing origami folds, and proving the correctness of origami constructions. Eos supports Huzita's fold operations and has been extended to include multi-fold operations. We use Eos, to assist us with our mathematical study of the knot fold.

The rest of the paper is organized as follows. In Sect. 2, we give the notations that we use. In Sect. 3, we present Huzita's fold operations and their extensions. In Sect. 4, we explain the geometrical properties of the knot fold. The construction of regular heptagon by Eos is shown in Sect. [5, and its formal and algebraic proof is discussed in Sect. 6. In Sect. 7, we summarize our results and point out a direction of further research.

\section{Notations}

In this paper, we restrict the use of geometrical objects to points, segments and lines. Points are denoted by a single capital letter of the Latin alphabet, e.g. $A, B, C, D, X, Y$ etc. Lines are denoted by $m, n, t, u$ and $v$. Let $X$ and $Y$ be two points, then $\overline{X Y}$ denotes the line passing through points $X$ and $Y$. For brevity, we often write $X Y$ to refer to the line passing through $X$ and $Y$. We use the same notation to denote the segment between points $X$ and $Y$. Although the meaning of the notation $X Y$ should be clear from the context, we precede the notation $X Y$ with either the word "segment" or the word "line" to emphasize which objects we are working with. We also use $\overrightarrow{X Y}$ to denote a vector from point $X$ to point $Y$. The distance between two points $X$ and $Y$ is denoted by $|X Y|$.

Since we use Cartesian coordinate system in this paper, a line is represented by a linear equation $a x+b y+c=0$ in variables $x$ and $y$. The sets of all points and lines are denoted by $\Pi$ and $\mathcal{L}$, respectively. Abusing the set notation, we use $X \in m$ to mean that point $X$ is incident to line $m$, and $\left\{X_{1}, \ldots, X_{p}\right\} \subset m$ to mean that all the points $X_{1}, \ldots, X_{p}$ are incident to $m$.

When an origami is folded along a line $m$, some of the points are moved to superpose with their reflections across $m$. We denote by $X^{m}$ the reflection of point $X$ across line $m$.

A simple knot is denoted by $\mathcal{K} . \mathcal{K}_{i}$ denotes the $i$ th knot. The notation $\mathcal{K}=\langle m, n, t\rangle$ means that the knot $\mathcal{K}$ is defined by 3 fold lines (to be explained in Sect. 4) $m, n$ and $t$, and furthermore $\mathcal{K}$ is obtained by folding along the lines following the order of their appearance in $\langle m, n, t\rangle$.

\section{Origami Geometrical Construction}

\subsection{Huzita's Fold Operations}

By $\mathcal{O}$ we denote an origami. An origami $\mathcal{O}$ is supposed to represent a square sheet of paper with four points on the corners and four edges that is subject to folding ${ }^{1}$. We call $A, B, C$ and $D$, the points on the corner. Some intersections of lines may not fit on the square paper.

\footnotetext{
${ }^{1}$ We could take $\mathcal{O}$ to be any convex polygon that can be constructed from a square sheet of paper. However, this could be an unnecessary generalization in our study.
} 
As we want to work with these points, we consider $\mathcal{O}$ to be a sufficiently large (bounded) 2D plane so that all the points and lines of interest are on $\mathcal{O}$. Huzita observed that the degree of freedom of paper fold by fold lines can be made finite by specifying how certain points and lines are superposed. Then, he gave the following operations $(\mathrm{O} 1) \sim(\mathrm{O} 6)$, which serve as basic operations in the geometrical construction of origamis. Operation (O7) was added, later, by Justin [6]. We call collectively $(\mathrm{O} 1) \sim(\mathrm{O} 7)$ Huzita's fold operations.

(O1) Given two distinct points $P$ and $Q$, fold $\mathcal{O}$ along the unique line that passes through $P$ and $Q$.

(O2) Given two distinct points $P$ and $Q$, fold $\mathcal{O}$ along the unique line to superpose $P$ and $Q$.

(O3) Given two distinct lines $m$ and $n$, fold $\mathcal{O}$ along a line to superpose $m$ and $n$.

(O4) Given a line $m$ and a point $P$, fold $\mathcal{O}$ along the unique line passing through $P$ to superpose $m$ onto itself.

(O5) Given a line $m$, a point $P$ not on $m$ and a point $Q$, fold $\mathcal{O}$ along a line passing through $Q$ to superpose $P$ and $m$.

(O6) Given two lines $m$ and $n$, a point $P$ not on $m$ and a point $Q$ not on $n$, where $m$ and $n$ are distinct or $P$ and $Q$ are distinct, fold $\mathcal{O}$ along a line to superpose $P$ and $m$, and $Q$ and $n$.

(O7) Given two lines $m$ and $n$ and a point $P$ not on $m$, fold $\mathcal{O}$ along the unique line to superpose $P$ and $m$, and $n$ onto itself.

We note that the above statements are slightly different from the original ones. To formalize the above statements with the view to rigorous geometrical construction, we restate Huzita's operations by carefully adding and removing side conditions of degeneracy and incidence [7. In essence, treating origamis with a program requires rigorous specification of these operations.

Huzita's fold operations determine fold lines by specifying superpositions of constructed points and lines. In Eos, these specifications are formulas in a language of many-sorted firstorder predicate logic. By the algebraic interpretation of the formulas we derive polynomial equalities. The problem of finding fold line(s) is therefore reduced to solving constraints expressed in multi-variate polynomials of degree 3 over the field of origami constructible numbers [8, 5].

\subsection{Extensions}

The contribution of Huzita's fold operations is powerful enough to perform relevant geometrical constructions by way of solving algebraic equations of degree up to 3. Examples of such constructions are trisecting an arbitrary angle, constructing a regular heptagon, etc. Some studies have explored possible extensions of Huzita's fold operations in an attempt to increase the power of paper folding, i.e. to solve higher degree equations.

Alperin and Lang proposed multi-fold method, where the origami is folded along more than one fold line, simultaneously 9 . The idea is to find fold lines that are mutually dependent. The multi-fold construction allows solving higher degree equations. We presented a construction method of angle trisection using 2-fold operation [5] and angle quintisection using 4-fold operation [10. Although the $p$-fold method generates an arbitrarily high degree polynomial, accurately folding an origami by $p$ lines simultaneously would be difficult to do by hand even for $p=2$. 
Folding polygonal knots has been traditionally used in Japan. For example, folding a broad sash for a kimono by a pentagon is a common practice of daily life. However, it is unknown when the knot fold was first mathematically studied. The earliest contributions of which we are aware are those of Cundy and Rollett [11], Brunton [12] and Sakaguchi [13. Cundy and Rollett showed models of knots that make some regular polygons. Brunton elaborated the mathematical study of the knot fold and showed that the number of necessary knots depends on the number of the edges of the polygon.

\section{Knot Fold}

In order to determine the geometrical constraints of knot fold, we first analyze key geometrical properties of a knot.

\subsection{Geometrical Properties}

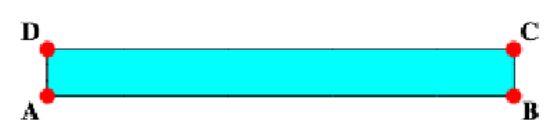

(a)

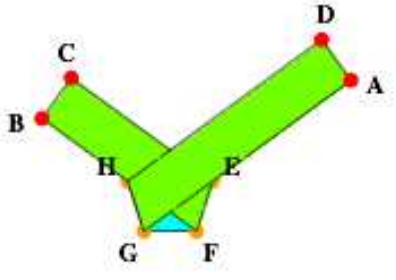

(b)

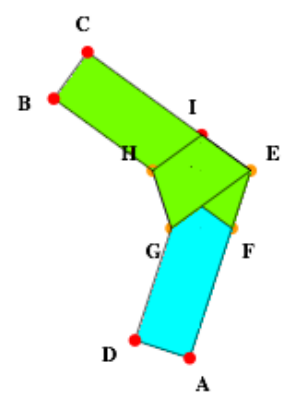

(c)

Figure 1: Knot-fold of regular pentagon FEIHG

Let us examine the operation of knotting in Fig. 1. We make one simple knot by tying together the two ends of the origami tape in Fig. 1(a) Note that we use a rectangular shape of an origami. When the height of the tape, i.e $|A D|$ and $|B C|$, is infinitesimal and both ends of the paper are connected, the tape becomes a curve, i.e. an object of study in knot theory. The knot with 3 crossings is the most basic one in knot theory. When we bring the height back to the original without distorting the tape, except for folds, we obtain the polygonal knot. A well fastened and well flattened knot becomes a polygonal shape as depicted in Fig. 1(c), The obtained polygonal shape exhibits a regular pentagonal form. As it is inferred from the knot theory, making the knot in Fig. 1(c) requires 3 folds along the lines $m, n$ and $t$ that extend the edges $F E, G H$ and $I E^{n}$, respectively. ${ }^{2}$ The knot $\mathcal{K}=\langle m, n, t\rangle$ is the one in Fig. 1(c).

When the knot is entirely unfolded, we obtain the tape with the creases and the generated points as shown in Fig. 2. We note the following geometrical properties. The vertices $E, H$ and $I$ are lined up on the edge $C D$ whereas vertices $F$ and $G$ are incident to the edge $A B$. The fold along $m$ passes through $F$ and superposes point $H$ and line $A B$. Point $E$ is the intersection of $m$ and $C D$. Similarly, the fold line $n$ passes through $G$, and the fold along $n$ superposes point $E$ and line $A B$. Point $H$ is the intersection of $n$ and $C D$. Note that fold lines $m$ and $n$ are mutually defined. The fold is a 2-fold operation where $m$ and $n$ are defined simultaneously. Line $t$ can be determined by applying operation (O5) of Huzita's fold operation set. Namely,

\footnotetext{
${ }^{2}$ Recall that $E^{n}$ is the reflection point of $E$ across line $n$ as defined in Sect. 2
} 


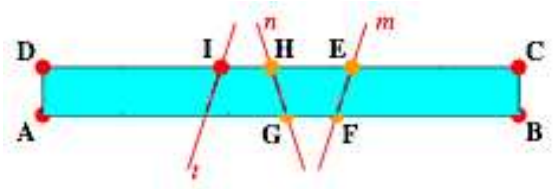

Figure 2: Unknotted tape

line $t$ passes through $I$ and superposes $G$ and $C D$. Note that the parameters of (O5), points $I$, $G$ and line $C D$ are considered with respect to the configuration before the fold. Refer to Fig. 2, The origami after the the 2-fold (i.e. along $m$ and $n$ ) is shown in Fig. 1(b). The purposes of the fold along $t$ is, first, to construct the vertex $I$ and, second, to fasten and lock the knot.

A purely geometrical proof that the knot fold creates a regular polygon is sketched in [14. In the following, we present a formal and automated algebraic proof that uses properties of the operation of folding such as preservation of some of distances, parallelism, etc.

\subsection{Geometrical Constraint Solving Approach}

The question now becomes how to determine the fold lines $m, n$ and $t$ such that the knot is a regular pentagon. The proof that the knot fold gives rise to regular pentagons shows that each of the fold line makes angles $2 \alpha=\frac{2 \pi}{5}$ with the edges [14, where $\alpha=\measuredangle G E H=\measuredangle E H F$. In origami geometry, construction of angles using Huzita's fold operations is not trivial. The problem of constructing angles is boiled down to a sequence of fold steps which makes the construction tedious where the number of intermediate points and lines is prone to increase. Furthermore, not all angles are constructible by Huzita's fold operations. The construction of a regular 11-gon, whose interior angles are equal to $\frac{9 \pi}{11}$, is shown to be impossible by Huzita's fold operations [15]. Hence, we carefully choose a specification of the knot fold that could be extended towards the construction of regular $n$-gons, where $n \geq 7$. We are led to avoid construction of angles in order to solve the knot fold construction problem. We consider the knot fold construction as a geometrical constraint solving problem without use of angles.

\section{Example: Regular pentagon}

Our method of constructing a regular pentagon by the knot fold uses a multi-fold together with Huzita's fold operations. We consider the example in Fig. 1 where we make a knot $\mathcal{K}=\langle m, n, t\rangle$. We try to define the geometrical properties of $\mathcal{K}$ in a systematic way so that we can generalize it to the knot fold of regular $n$-gons, where $n \geq 7$. We observe the following geometrical properties on $m, n, t, E, F, G, H$ and $I$.

- Points $E$ and $F$ are incident to $m$.

- Points $G$ and $H$ are incident to $n$.

- Points $E, H$ and $I$ are incident to $C D$.

- Points $F$ and $G$ are incident to $A B$.

- The fold line $m$ superposes $H$ and $A B$, i.e. $H^{m} \in A B$.

- The fold line $n$ superposes $E$ and $A B$, i.e. $E^{n} \in A B$.

- The fold line $t$ passes through $I$ and superposes $G$ and $C D$. i.e. $I \in t$ and $G^{t} \in C D$.

- The distances $|E F|,|F G|$ and $|G H|$ are equal. 
The above properties are described by the following first order logical formula $\phi_{\mathcal{K}}$.

$$
\begin{aligned}
\phi_{\mathcal{K}} \equiv & \exists m, n, t \in \mathcal{L} \exists E, F, G, H, I \in \Pi \\
& \{E, F\} \subset m \wedge\{G, H\} \subset n \wedge\{E, H, I\} \subset C D \wedge\{F, G\} \subset A B \wedge \\
& H^{m} \in A B \wedge E^{n} \in A B \wedge I \in t \wedge G^{t} \in C D \wedge|E F|=|F G|=|G H|
\end{aligned}
$$

We used Eos to construct the regular pentagon in Fig. 1. It is possible to determine $t$ independently from $m$ and $n$. We consider the formula (2), that defines the 2-fold operation to solve lines $m$ and $n$, first.

$$
\begin{aligned}
\phi_{\mathcal{K}}^{\prime} \equiv & \exists m, n \in \mathcal{L} \exists E, F, G, H \in \Pi \\
& \{E, F\} \subset m \wedge\{G, H\} \subset n \wedge\{E, H\} \subset C D \wedge\{F, G\} \subset A B \wedge \\
& H^{m} \in A B \wedge E^{n} \in A B \wedge|E F|=|F G|=|G H|
\end{aligned}
$$

The fold line $t$ is obtained by applying operation (O5). Namely, line $t$ passes through point $I$ and superposes point $H$ and line $A B$.

We can prove automatically that the generated shape is a regular pentagon based on Gröbner bases theory. We will omit the proof of the correctness of the pentagon knot construction. In the next sections, we will discuss the construction and the proof of a regular heptagon by the knot fold in details.

\section{Regular Heptagon by the Knot Fold}

Brunton studied the construction of regular $n$-gons, where $n \geq 3$ [12]. He showed that the number of necessary knots is $\frac{\Phi(n)}{2}-1$ in order to construct a regular $n$-gon, where $\Phi(n)$ is Euler's totient function. Hence, to construct a regular heptagon, we perform 2 knots $\mathcal{K}_{1}$ and $\mathcal{K}_{2}$. We explained in Sect. 4 that a knot can be decomposed into 3-fold operations along three fold lines. In the case of regular heptagon, we need 5 fold lines $m, n, t, u$ and $v$, where $\mathcal{K}_{1}=\langle m, n, t\rangle$ and $\mathcal{K}_{2}=\langle v, u, m\rangle$. Let EKJIHGF be the constructed regular heptagon. Figure 3 exhibits the vertices of the regular heptagon after unfolding $\mathcal{K}_{1}$ and $\mathcal{K}_{2}$. The fold lines are extensions of edges of EKJIHGF as follows. Lines $m, n$ and $t$ are the extension of segment $E F, I J$ and $G F^{n}$, respectively. Lines $v$ and $u$ are extensions of segments $K E^{u}$ and $H I^{m}$, respectively. Figure 4 shows a sketch of a regular heptagon with the lines $m, n, t, u$ and $v$.

\subsection{Geometrical Constraints}

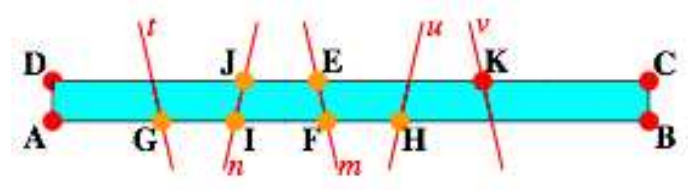

Figure 3: Unfolded regular heptagon EKJIHGF

Lines $m$ and $n$ of the knot $\mathcal{K}_{1}=\langle m, n, t\rangle$ are defined by the following properties on $m, n, t$, $E, F, I, J, G$ and $H$. 


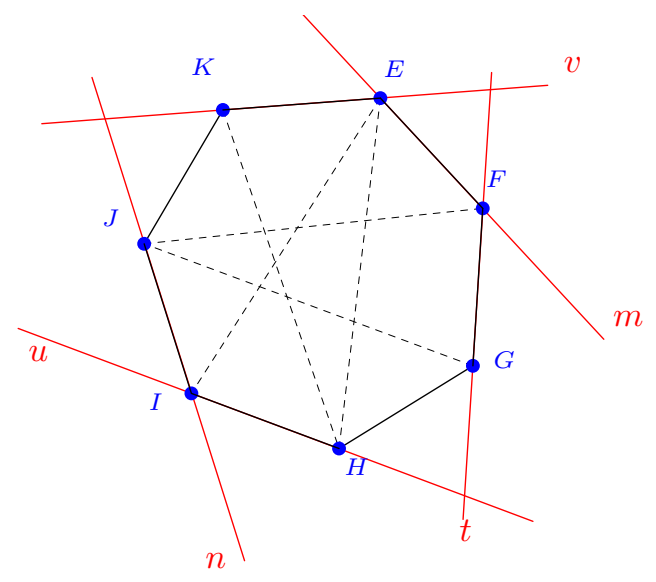

Figure 4: Sketch of a regular heptagon EKJIHGF and lines $m, n, t, u$ and $v$

- Points $E$ and $F$ are incident to $m$.

- Points $I$ and $J$ are incident to $n$.

- Points $E$ and $J$ are incident to $C D$.

- Points $F, I, G$ and $H$ are incident to $A B$.

- The fold line $m$ superposes $I$ and $C D$, i.e. $I^{m} \in C D$.

- The fold line $n$ superposes $F$ and $C D$, i.e. $F^{n} \in C D$.

- The distances $|E F|,|I J|,\left|F G^{n}\right|,\left|I H^{m}\right|$ and $\left|G^{n} H^{m}\right|$ are equal. ${ }^{3}$

We write formula $\phi_{\mathcal{K}_{1}}$ in a similar fashion to the formula (1).

$$
\begin{aligned}
\phi_{\mathcal{K}_{1}} \equiv & \exists m, n, t \in \mathcal{L} \exists E, F, I, J, G, H \in \Pi \\
& \{E, F\} \subset m \wedge\{I, J\} \subset n \wedge\{F, I, G, H\} \subset A B \wedge\{E, J\} \subset C D \wedge \\
& I^{m} \in C D \wedge F^{n} \in C D \wedge G \in t \wedge J^{t} \in A B \wedge \\
& |E F|=|I J|=\left|F G^{n}\right|=\left|I H^{m}\right|=\left|G^{n} H^{m}\right|
\end{aligned}
$$

Similarly to our discussion in Sect.4.2 line $t$ can be constructed independently from lines $m$ and $n$. We therefore can separate the construction $t$ and use $\phi_{\mathcal{K}_{1}}^{\prime}$ in (4) to solve for $m$ and $n$, first.

$$
\begin{aligned}
\phi_{\mathcal{K}_{1}}^{\prime} \equiv & \exists m, n \exists E, F, I, J, G, H \in \Pi \\
& \{E, F\} \subset m \wedge\{I, J\} \subset n \wedge\{F, I, G, H\} \subset A B \wedge\{E, J\} \subset C D \wedge \\
& I^{m} \in C D \wedge F^{n} \in C D \wedge \\
& |E F|=|I J|=\left|F G^{n}\right|=\left|I H^{m}\right|=\left|G^{n} H^{m}\right|
\end{aligned}
$$

Knot $\mathcal{K}_{2}=\langle v, u, m\rangle$ is defined by the following properties on $v, u, m, K, X, H, Y, Z$ and $F$.

- Points $K$ and $X$ are on $v$.

- Points $H$ and $Y$ are on $u$.

- Points $K$ and $Y$ are on $C D$.

- Points $Z, H, F$ and $X$ are on $A B$.

- The fold line $v$ superposes $H$ and $C D$, i.e. $H^{v} \in C D$.

\footnotetext{
${ }^{3}$ Refering to Fig. 3 distances $\left|G F^{n}\right|$ and $\left|F G^{n}\right|$ are equal due to the fact that reflection across line n preserves the distances. Similarly, distances $\left|H I^{m}\right|$ and $\left|I H^{m}\right|$ are equal.
} 
- The fold line $u$ superposes $X$ and $C D$, i.e. $X^{u} \in C D$.

- The fold line $m$ passes through $F$ and superposes $Y$ and $A B$, i.e. $Y^{m} \in A B$.

- The distances $|K X|,|H Y|,\left|X F^{u}\right|,\left|H Z^{v}\right|$ and $\left|F^{u} Z^{v}\right|$ are equal.

$$
\begin{aligned}
\phi_{\mathcal{K}_{2}} \equiv & \exists v, u, m \in \mathcal{L} \exists K, X, H, Y, Z, F \in \Pi \\
& \{K, X\} \subset v \wedge\{H, Y\} \subset u \wedge\{K, Y\} \subset C D \wedge\{X, H, Z, F\} \subset A B \wedge \\
& H^{v} \in C D \wedge X^{u} \in C D \wedge F \in m \wedge Y^{m} \in A B \wedge \\
& |K X|=|H Y|=\left|X F^{u}\right|=\left|H Z^{v}\right|=\left|F^{u} Z^{v}\right|
\end{aligned}
$$

However, referring to Fig. 3. points $X, Y$ and $Z$ are equal to points $E^{u}, I^{m}$ and $\left(\left(\left(\left(G^{n}\right)^{m}\right)^{u}\right)^{v}\right)$. From formula $\phi_{\mathcal{K}_{1}}^{\prime}, E, I$ and $G$ are obtained. It is possible to determine the fold lines that make $\mathcal{K}_{2}$ in an easier way than solving constraints in formula (5), as we explain in the next sub-section.

\subsection{Construction by Eos}

We first assume that the initial origami is a rectangle $A B C D$. We work with the Cartesian coordinate system, and the coordinates of points $A, B, C$ and $D$ are $(0,0),(w d, 0),(w d, h t)$ and $(0, h t)$, respectively. The width $w d$ and the height $h t$ can be taken arbitrary. Of course $w d$ is sufficiently larger than $h t$, so that the knot construction is feasible. For concreteness of our presentation using Eos, we set $w d=900$ and $h t=60$. Furthermore, let $E$ be an arbitrary but fixed point on segment $C D$. Let the coordinates of $E$ be $(400$, ht) for simplicity and clarity of the construction. Our objective is to construct a regular heptagon $E K J I H G F$ by the knot fold. Recall that the construction requires a 2-fold operation prior to Huzita's fold operations (see Sect. 4.1). The first folding step is the crucial one, i.e. the 2-fold operation.

In Eos, Huzita's fold operations can be performed using function HO. The extension to the multi-fold is natural as $\mathrm{HO}$ is implemented with the generality that allows the specification of logical formulas describing the multi-fold. Multi-fold is realized by the call of the following Mathematica function.

$$
\text { HO }[\mathcal{H} \text {, Constraint } \rightarrow \phi]
$$

$\mathcal{H}$ is a list of points on the origami which determine the faces to be moved. $\phi$ is a formula in the first-order predicate logic. The formula $\phi$ specifies the constraints that the geometrical objects concerned have to satisfy. In our example, the geometrical constraints that specify the 2 -fold operation are expressed by formula $\phi_{\mathcal{K}_{1}}^{\prime}$ in (4). We write $\phi_{\mathcal{K}_{1}}^{\prime}$ in the language of Eos and call HO as follows.

$$
\begin{aligned}
\mathrm{HO}[\{\mathrm{C}, \mathrm{A}\}, & \text { Constraint } \rightarrow \exists_{m, m: \operatorname{Line}} \exists_{n, n: \operatorname{Line}} \exists_{f, f: \text { Point }} \exists_{i, i: \text { Point }} \exists_{j, j: \text { Point }} \exists_{g, g: \text { Point }} \exists_{h, h \text { :Point }} \\
& (\{\mathrm{E}, f\} \subset m \wedge\{i, j\} \subset n \wedge\{f, i, g, h\} \subset \mathrm{AB} \wedge j \in \mathrm{CD} \wedge \\
& i^{m} \in \mathrm{CD} \wedge f^{n} \in \mathrm{CD} \wedge \\
& \text { SqDistance }[\mathrm{E}, f]==\text { SqDistance }[i, j]==\text { SqDistance }\left[f, g^{n}\right]== \\
& \text { SqDistance } \left.\left.\left[i, h^{m}\right]==\text { SqDistance }\left[g^{n}, h^{m}\right]\right)\right]
\end{aligned}
$$

The term SqDistance $[X, Y]$ is the square of the distance between $X$ and $Y$ (i.e. $|X Y|^{2}$ ). Notations like " $\exists_{m, m: L i n e}$ ", " $\{\mathrm{E}, f\} \subset m$ ", " $i^{m}$ ", " $i^{m} \in \mathrm{CD}$ " are Eos extension of Mathematica syntax.

The evaluation of (6) generates 24 distinct cases of possible configurations of points $F, G$, $H, I$ and $J$ and lines $m$ and $n$. We deduce that the algebraic interpretation of the constraints, as defined by $\phi_{\mathcal{K}_{1}}^{\prime}$, is a well constrained system of equations since we obtained finite number of solutions. However, not all of the 24 cases are relevant to our construction problem. They may 


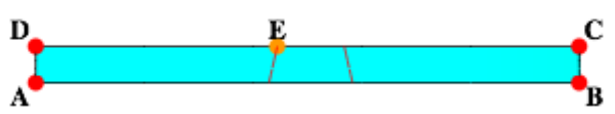

(a) Case 1

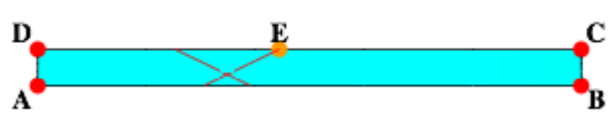

(c) Case 3

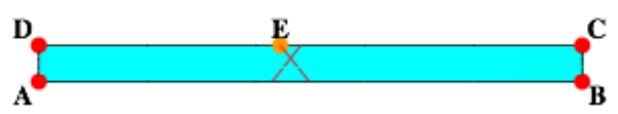

(e) Case 5

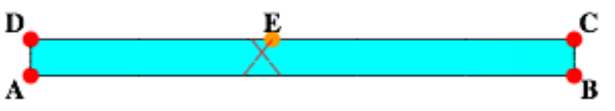

(b) Case 2

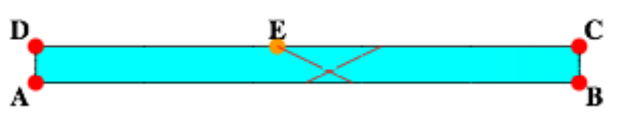

(d) Case 4

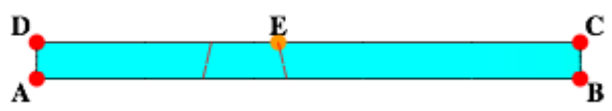

(f) Case 6

Figure 5: 6 possible cases for lines $m$ and $n$

correspond to degenerate cases that lead to failure of the proof by Gröbner bases. In order to eliminate the degenerate cases and also to get a reasonable number of cases, we further narrow the search space of the configuration of points and lines. For that, we add the following extra constraints.

$E \notin n \wedge j \notin m \wedge$ SqDistance $[E, i]==\operatorname{SqDistance}[j, f]==\operatorname{SqDistance}[E, h]==\operatorname{SqDistance}[j, g]$

The sub-formula $\mathrm{E} \notin n \wedge j \notin m$ eliminates the degenerate cases where $E$ and $j$ are equal. Sub-formula SqDistance $[E, i]==\operatorname{SqDi}$ tance $[j, f]==\operatorname{SqDi}$ tance $[E, h]==$ SqDistance $[j, g]$ specifies further conditions on points $E, F, G, H, I$ and $J$. We evaluate the following HO call.

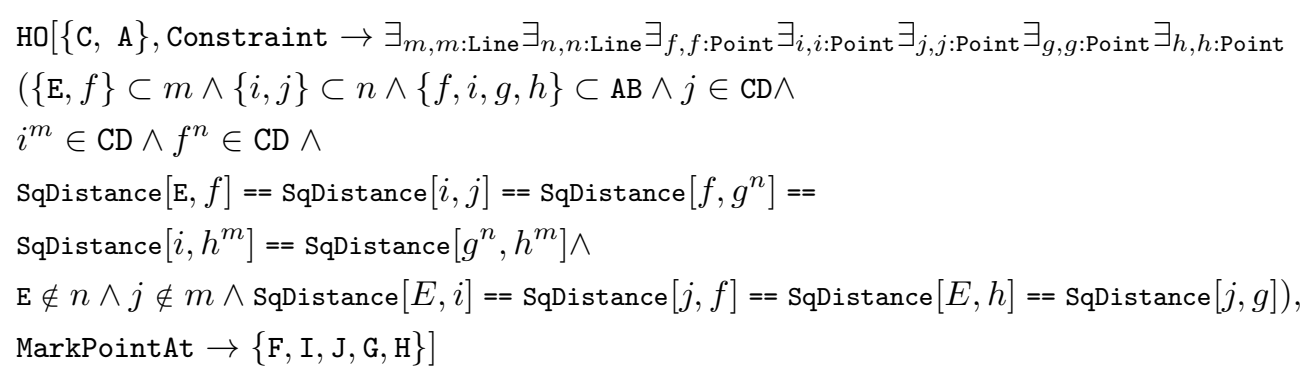

Consequently, we obtain 6 cases as shown in Fig. 15. Two of them, namely those in Fig. 5(a) and in Fig. 5(f)] lead to the construction of a regular heptagon. Eos allows the user to interactively choose the suitable case to proceed with the construction. Keyword MarkPointAt tells the Eos how to label the existentially quantified points $f, i, j, g$ and $h$. The outcome of folding in the case 6 is shown in Fig. 6

Next, we apply two operations (O1) to fold along the line $F G^{n}$ and $I H^{m}$ in Fig. 6 by calling HO with suitable arguments. The lines $F G^{n}$ and $I H^{m}$ are the fold lines $t$ and $u$, respectively. The results are shown in Fig. 7(a) and 7(b), Finally, given the origami of Fig. 7(b) we construct the remaining vertex $K$. We apply operation (O6) to obtain $v$, which brings $H$ and $G$ onto $I C$ and $H B$, respectively. Point $K$ is the intersection of $I C$ and line $v$. We obtain the heptagon EKJIHGF in Fig. 7(c) 


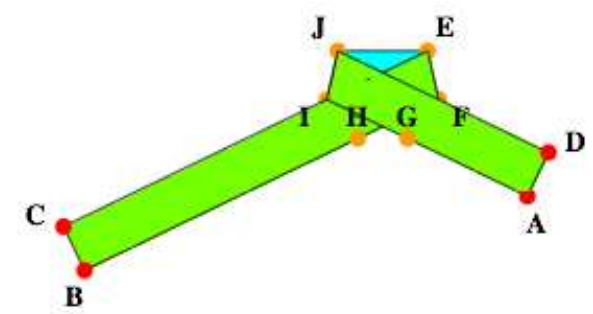

Figure 6: Step 1: Folds along lines $m$ and $n$

\subsection{Algebraic Interpretation}

This algebraic interpretation is used to "evaluate" the function calls of $\mathrm{HO}$ as well as the automated proof of the correctness of the construction. We take HO in (7) as an example. To obtain geometrical objects $m, n, F, G, H, I$ and $J$, the formula in (7) is transformed into a set of algebraic equalities. Further details of the algebraic interpretation are explained in 8 ] and [5].

Let the coordinates of $A, B, D$ and $E$ be $(0,0),(900,0),(0,60)$ and $(400,60)$, respectively. For an atomic formula $\phi$, let $\llbracket \phi \rrbracket$ denote the set of polynomial relations that are the algebraic meaning of $\phi$. An atomic formula is interpreted as a set of polynomial relations (equalities or inequalities), and a term is given as a rational function. The set of (non-simplified) polynomial equalities (8) - (14) is the algebraic interpretation of formula in (77).

$$
\begin{aligned}
& \{400 \mathrm{a} 5+60 \mathrm{~b} 6+\mathrm{c} 7==0, \mathrm{c} 7+\mathrm{a} 5 \mathrm{x} 11+\mathrm{b} 6 \mathrm{y} 12==0, \mathrm{c} 10+\mathrm{a} 8 \mathrm{x} 13+\mathrm{b} 9 \mathrm{y} 14==0, \\
& \mathrm{c} 10+\mathrm{a} 8 \mathrm{x} 15+\mathrm{b} 9 \mathrm{y} 16==0,-900 \mathrm{y} 12==0,-900 \mathrm{y} 14==0, \\
& -900 \mathrm{y} 20==0,-900 \mathrm{y} 18==0,-54000+900 \mathrm{y} 16==0 \\
& -54000+\frac{900\left(-2 \mathrm{~b} 6(\mathrm{c} 7+\mathrm{a} 5 \mathrm{x} 13)+\mathrm{a} 5^{2} \mathrm{y} 14-\mathrm{b} 6^{2} \mathrm{y} 14\right)}{\mathrm{a} 5^{2}+\mathrm{b} 6^{2}}==0, \\
& -54000+\frac{900\left(-2 b 9(\mathrm{c} 10+\mathrm{a} 8 \mathrm{x} 11)+\mathrm{a} 8^{2} \mathrm{y} 12-\mathrm{b} 9^{2} \mathrm{y} 12\right)}{\mathrm{a} 8^{2}+\mathrm{b}^{2}}==0, \\
& (400-\mathrm{x} 11)^{2}+(60-\mathrm{y} 12)^{2}==(\mathrm{x} 13-\mathrm{x} 15)^{2}+(\mathrm{y} 14-\mathrm{y} 16)^{2}== \\
& \left(\mathrm{y} 12-\frac{-2 \mathrm{~b} 9(\mathrm{c} 10+\mathrm{a} 8 \mathrm{x} 17)+\mathrm{a} 8^{2} \mathrm{y} 18-\mathrm{b} 9^{2} \mathrm{y} 18}{\mathrm{a} 8^{2}+\mathrm{b} 9^{2}}\right)^{2}+ \\
& \left(\mathrm{x} 11-\frac{-\mathrm{a} 8^{2} \mathrm{x} 17+\mathrm{b} 9^{2} \mathrm{x} 17-2 \mathrm{a} 8(\mathrm{c} 10+\mathrm{b} 9 \mathrm{y} 18)}{\mathrm{a} 8^{2}+\mathrm{b} 9^{2}}\right)^{2}== \\
& \left(\mathrm{y} 14-\frac{-2 \mathrm{~b} 6(\mathrm{c} 7+\mathrm{a} 5 \mathrm{x} 19)+\mathrm{a} 5^{2} \mathrm{y} 20-\mathrm{b} 6^{2} \mathrm{y} 20}{\mathrm{a} 5^{2}+\mathrm{b} 6^{2}}\right)^{2}+ \\
& \left(\mathrm{x} 13-\frac{-\mathrm{a} 5^{2} \mathrm{x} 19+\mathrm{b} 6^{2} \mathrm{x} 19-2 \mathrm{a} 5(\mathrm{c} 7+\mathrm{b} 6 \mathrm{y} 20)}{\mathrm{a} 5^{2}+\mathrm{b} 6^{2}}\right)^{2}== \\
& \left(\frac{-2 \mathrm{~b} 9(\mathrm{c} 10+\mathrm{a} 8 \mathrm{x} 17)+\mathrm{a} 8^{2} \mathrm{y} 18-\mathrm{b} 9^{2} \mathrm{y} 18}{\mathrm{a} 8^{2}+\mathrm{b} 9^{2}}-\frac{-2 \mathrm{~b} 6(\mathrm{c} 7+\mathrm{a} 5 \mathrm{x} 19)+\mathrm{a} 5^{2} \mathrm{y} 20-\mathrm{b} 6^{2} \mathrm{y} 20}{\mathrm{a} 5^{2}+\mathrm{b} 6^{2}}\right)^{2}+ \\
& \left(\frac{-\mathrm{a} 8^{2} \mathrm{x} 17+\mathrm{b} 9^{2} \mathrm{x} 17-2 \mathrm{a} 8(\mathrm{c} 10+\mathrm{b} 9 \mathrm{y} 18)}{\mathrm{a} 8^{2}+\mathrm{b} 9^{2}}-\frac{-\mathrm{a} 5^{2} \mathrm{x} 19+\mathrm{b} 6^{2} \mathrm{x} 19-2 \mathrm{a} 5(\mathrm{c} 7+\mathrm{b} 6 \mathrm{y} 20)}{\mathrm{a} 5^{2}+\mathrm{b} 6^{2}}\right)^{2},
\end{aligned}
$$




$$
\begin{array}{r}
400 \mathrm{a} 8+60 \mathrm{~b} 9+\mathrm{c} 10 \neq 0, \mathrm{c} 7+\mathrm{a} 5 \mathrm{x} 15+\mathrm{b} 6 \mathrm{y} 16 \neq 0, \\
(-400+\mathrm{x} 13)^{2}+(-60+\mathrm{y} 14)^{2}== \\
(\mathrm{x} 11-\mathrm{x} 15)^{2}+(\mathrm{y} 12-\mathrm{y} 16)^{2}==(-400+\mathrm{x} 19)^{2}+(-60+\mathrm{y} 20)^{2}== \\
(\mathrm{x} 15-\mathrm{x} 17)^{2}+(\mathrm{y} 16-\mathrm{y} 18)^{2}, \\
(-1+\mathrm{b} 9) \mathrm{b} 9=\mathrm{x}=0,(-1+\mathrm{a} 8)(-1+\mathrm{b} 9)=0,1+\mathrm{a} 8^{2} \neq 0,(-1+\mathrm{b} 6) \mathrm{b} 6==0 \\
\left.(-1+\mathrm{a} 5)(-1+\mathrm{b} 6)==0,1+\mathrm{a} 5^{2} \neq 0\right\}
\end{array}
$$

A line $a x+b y+c=0$ is represented by $(a, b, c)$, together with the constraint $(-1+b) b=$ $0 \wedge(-1+a)(-1+b)=0 \wedge a^{2}+1 \neq 0$. Lines $m$ and $n$ are represented by $(a 5, b 6, c 7)$ and $(a 8, b 9, c 10)$, respectively. Hence, we have the equalities and disequalities in (14).

The equalities in (8) are the algebraic interpretation of the sub-formula $\{E, f\} \subset m \wedge\{i, j\} \subset$ $n \wedge\{f, i, g, h\} \subset A B \wedge j \in C D$. The first equation $400 a 5+60 b 6+c 7==0$ means that point $E$ at $(400,60)$ is incident to $m$ defined by the equation $a 5 x+b 6 y+c 7=0$. Similarly, the rest of the equations in (88) are interpretations of $f$ at $(x 11, y 12)$ is on $m, i$ at $(x 13, y 14)$ is on $n, j$ at $(x 15, y 16)$ is on $n, f$ is on $A B, i$ is on $A B, h$ at $(x 19, y 20)$ is on $A B, g$ at $(x 17, y 18)$ is on $A B$ and $j$ is on $C D$, respectively.

The reflection of point $i$ at $(x 13, y 14)$ across line $m$ is the point $i^{m}$ whose coordinates are

$$
\left(\frac{-a 5^{2} x 13+b 6^{2} x 13-2 a 5(c 7+b 6 y 14)}{a 5^{2}+b 6^{2}}, \frac{-2 b 6(c 7+a 5 x 13)+a 5^{2} y 14-b 6^{2} y 14}{a 5^{2}+b 6^{2}}\right)
$$

Equation (9) states that $i^{m}$ is incident to $C D$ represented by $(0,1,-60)$. Similarly, equation (10) is the algebraic interpretation of $f^{n} \in C D$. Eos transforms rational form $\frac{p}{q}==0$ to $p==0$. Note that in (9)-(11), $q$ comes from coefficient of lines and $q \neq 0$ is deduced from (14).

The disequalities of (12) states that the coordinates of point $E$ and $j$ do not satisfy the equation of lines $n$ and $m$, respectively. Eos changes the disequalities into equalities by adding slack variables introduced by Rabinowitch trick.

Now, we examine the equalities (11) and (13). 【SqDistance $[E, f]==$ SqDistance $[i, j] \rrbracket$ gives rise to the first equality in (11), namely $(400-x 11)^{2}+(60-y 12)^{2}==(x 13-x 15)^{2}+(y 14-y 16)^{2}$, where $\mathrm{E}$ at $(400,60), f$ at $(x 11, y 12), i$ at $(x 13, y 14)$ and $j$ at $(x 15, y 16)$. The rest of the equalities in (11) and equalities in (13) are obtained in the same way.

By solving the above set of polynomial equalities for the coefficients of $m$ and $n$ and coordinates of $f, i, j, g$ and $h$, we obtain the 6 cases in Fig. 5 .

\section{Proof}

\subsection{Theorem to Prove}

We prove the following theorem.

Theorem 6.1. Given the origami in Fig. 7(c), we have

(a) $|E F|=|F G|=|G H|=|H I|=|I J|=|J K|=|K E|$, and

(b) $\measuredangle E O F=\measuredangle F O G=\measuredangle G O H=\measuredangle H O I=\measuredangle I O J=\measuredangle J O K=\measuredangle K O E=\frac{2 \pi}{7}$, where $O$ is the center of EKJIHGF.

Let $\theta=\measuredangle E O F$ and $\alpha=\mathrm{e}^{i \theta}$. Vector $\overrightarrow{F G}$ is the rotation of $\overrightarrow{E F}$ through $\theta$ around center $O$. To prove the theorem 6.1 , we show that the rotations of $\overrightarrow{E F}$ through angles $2 \theta, 3 \theta, 4 \theta, 5 \theta$, and 


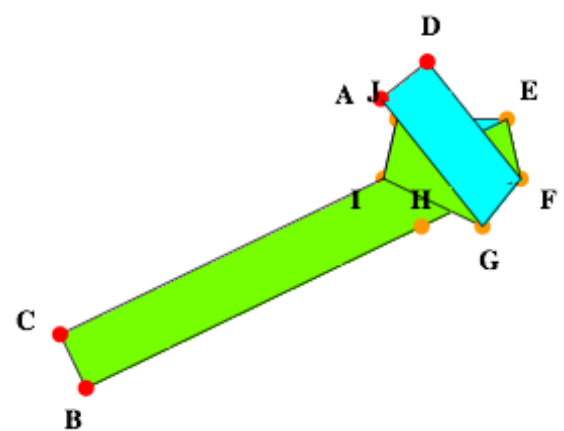

(a)

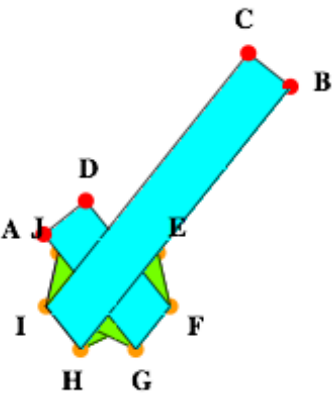

(b)

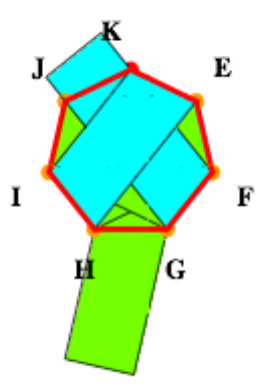

(c)

Figure 7: Construction of (a) the edge $G F$ (step 2), (b) the edge $I H$ (step 3) and (c) vertex $K$ and final heptagon $E K J I H G$ (step 4)

$6 \theta$ gives $\overrightarrow{G H}, \overrightarrow{H I}, \overrightarrow{I J}, \overrightarrow{J K}$ and $\overrightarrow{K E}$, respectively, and furthermore, that $\theta=\frac{2 \pi}{7}$. We show that after the construction, the following holds.

$$
\begin{aligned}
\forall \alpha \in \mathbb{C} \quad(\alpha \overrightarrow{E F}-\overrightarrow{F G}=0 & \Rightarrow \\
& \alpha^{2} \overrightarrow{E F}-\overrightarrow{G H}=0 \wedge \alpha^{3} \overrightarrow{E F}-\overrightarrow{H I}=0 \wedge \alpha^{4} \overrightarrow{E F}-\overrightarrow{I J}=0 \wedge \\
& \left.\alpha^{5} \overrightarrow{E F}-\overrightarrow{J K}=0 \wedge \alpha^{6} \overrightarrow{E F}-\overrightarrow{K E}=0 \wedge \alpha^{7}-1=0\right)
\end{aligned}
$$

Let $\mathcal{P}$ be the geometrical constraints accumulated during the construction. Let $\mathcal{C} \equiv \forall \alpha \in$ $\mathbb{C}\left(\mathcal{C}_{1} \Rightarrow \mathcal{C}_{2} \wedge \mathcal{C}_{3} \wedge \mathcal{C}_{4} \wedge \mathcal{C}_{5} \wedge \mathcal{C}_{6} \wedge \mathcal{C}_{7}\right)$ be the formula (15). $\mathcal{P}$ and $\mathcal{C}$ form the premise and conclusion of the proposition (16) that we want to prove.

$$
\mathcal{P} \Rightarrow \forall \alpha \in \mathbb{C} \quad\left(\mathcal{C}_{1} \Rightarrow \mathcal{C}_{2} \wedge \mathcal{C}_{3} \wedge \mathcal{C}_{4} \wedge \mathcal{C}_{5} \wedge \mathcal{C}_{6} \wedge \mathcal{C}_{7}\right)
$$

Formula (16) is semantically equivalent to the conjunctions of the formulas (17)-(22).

$$
\begin{aligned}
& \mathcal{P} \Rightarrow \forall \alpha \in \mathbb{C}\left(\mathcal{C}_{1} \Rightarrow \mathcal{C}_{2}\right) \\
& \mathcal{P} \Rightarrow \forall \alpha \in \mathbb{C}\left(\mathcal{C}_{1} \Rightarrow \mathcal{C}_{3}\right) \\
& \mathcal{P} \Rightarrow \forall \alpha \in \mathbb{C}\left(\mathcal{C}_{1} \Rightarrow \mathcal{C}_{4}\right) \\
& \mathcal{P} \Rightarrow \forall \alpha \in \mathbb{C}\left(\mathcal{C}_{1} \Rightarrow \mathcal{C}_{5}\right) \\
& \mathcal{P} \Rightarrow \forall \alpha \in \mathbb{C}\left(\mathcal{C}_{1} \Rightarrow \mathcal{C}_{6}\right) \\
& \mathcal{P} \Rightarrow \forall \alpha \in \mathbb{C}\left(\mathcal{C}_{1} \Rightarrow \mathcal{C}_{7}\right)
\end{aligned}
$$

\subsection{Proof by Eos}

We show how the validity of (17) is proved and the rest is achieved in a similar way. We add the conclusion $\forall \alpha \in \mathbb{C} \mathcal{C}_{1} \Rightarrow \mathcal{C}_{2}$ by calling Eos function Goal as follows.

$$
\begin{aligned}
\text { Goal }\left[\forall_{\alpha, \alpha \in \text { Complexes }}\right. & \text { VectorToComplex }[\alpha \overrightarrow{E F}-\overrightarrow{F G}]==0 \Rightarrow \\
& \left.\left.\left(\text { VectorToComplex }\left[\alpha^{2} \overrightarrow{E F}-\overrightarrow{G H}\right]==0\right)\right)\right]
\end{aligned}
$$

Expressions $\mathcal{C}_{1} \equiv \alpha \overrightarrow{E F}-\overrightarrow{F G}=0$ and $\mathcal{C}_{2} \equiv \alpha^{2} \overrightarrow{E F}-\overrightarrow{G H}=0$ written in the language of Eos are VectorToComplex $[\alpha \overrightarrow{E F}-\overrightarrow{F G}]$ and VectorToComplex $\left[\alpha^{2} \overrightarrow{E F}-\overrightarrow{G H}\right]==0$, respectively. 
Function Goal adds the negation of the conclusion to the premise. By calling Goal, we obtain $\mathcal{P} \wedge \neg\left(\forall \alpha \in \mathbb{C}\left(\mathcal{C}_{1} \Rightarrow \mathcal{C}_{2}\right)\right)$. In order to translate $\mathcal{P} \wedge \neg\left(\forall \alpha \in \mathbb{C}\left(\mathcal{C}_{1} \Rightarrow \mathcal{C}_{2}\right)\right)$ into algebraic form, we fix the coordinate system to be Cartesian with points $A, B, C, D$ and $E$ as follows:

$$
\begin{aligned}
\operatorname{map}=\operatorname{DefMapping}[\{\{\mathrm{A}, \text { Point }[0,-\mathrm{wd}]\},\{\mathrm{B}, \text { Point }[\mathrm{wd}, 0]\}, \\
\{\mathrm{C}, \text { Point }[\mathrm{wd}, \mathrm{ht}]\},\{\mathrm{D}, \text { Point }[0, \mathrm{ht}]\},\{\mathrm{E}, \text { Point }[0, \mathrm{ht}]\}\},\{\}] \&
\end{aligned}
$$

Without loss of generality, we set the size of the initial origami to be $2 w d \times h t$, where the width $w d$ is taken to be arbitrary and the height $h t$ is equal to 1 . The point $E$ is fixed at location $(0, h t)$. Finally, we check whether the reduced Gröbner basis of the algebraic interpretation of $\mathcal{P} \wedge \neg\left(\forall \alpha \in \mathbb{C}\left(\mathcal{C}_{1} \Rightarrow \mathcal{C}_{2}\right)\right)$ is $\{1\}$ by calling function Prove.

$$
\begin{gathered}
\text { Prove["Knot Heptagon", Mapping } \rightarrow \text { map, GroebnerBasis } \rightarrow \\
\{\text { CoefficientDomain } \rightarrow \text { RationalFunctions, } \\
\text { MonomialOrder } \rightarrow \text { DegreeReverseLexicographic }\}]
\end{gathered}
$$

The above call of function Prove tells Eos to compute Gröbner basis of the polynomial set $\llbracket \mathcal{P} \wedge \neg\left(\forall \alpha \in \mathbb{C}\left(\mathcal{C}_{1} \Rightarrow \mathcal{C}_{2}\right)\right) \rrbracket$. Let $\mathcal{V}$ be the set of variables in $\llbracket \mathcal{P} \wedge \neg\left(\forall \alpha \in \mathbb{C}\left(\mathcal{C}_{1} \Rightarrow \mathcal{C}_{2}\right)\right) \rrbracket$. The Gröbner basis computation is carried out in the domain of polynomials whose variables are in $\mathcal{V} \backslash\{\mathrm{wd}\}$ and whose coefficients are in functions of $\mathbb{Q}(\mathrm{wd})$. EOS computes Gröbner basis and generates a proof document (whose title is given by the first argument of the call of Prove) [16.

\subsection{Proof Results}

The proofs of (17)-(22) are successful. The CPU time used for Gröbner basis computation on Mac OS X (Intel Core i7 8G 2.4GHz) machine varies from 69.236984 seconds (for proving (17)) to 1994.889588 seconds (for proving (201)).

\section{Conclusion}

We presented the construction of a regular heptagon using the knot fold. Our method consists in applying 2-fold operation coupled with Huzita's fold operations. The fold lines that make the knot fold are determined by solving geometrical constraints. We further showed the proof of the correctness of the construction of a regular heptagon based on Gröbner bases.

Note that using Huzita's fold operations $(\mathrm{O} 1) \sim(\mathrm{O} 7)$, a regular heptagon is constructible. With Eos we need 9 Huzita's fold operations and more than 10 auxiliary operations such as unfolding and marking supporting points. Further investigation of the geometrical constraints by the knot fold is required to construct regular 11-gon, which is impossible by Huzita's fold operations.

Another issue that should be investigated in the future research is the rigidity of the knot fold. We need to define the notion of rigidity in mathematical terms in this knot fold as well as in more general origami that may involve $3 \mathrm{D}$ constructions.

\subsection{Acknowledgments}

We thank Stephen Watt of Western Ontario University for drawing our attention to the knot fold. This work is supported by JSPS KAKENHI Grant No. 25330007. The first author of this paper is supported by a postdoctoral fellowship at Kwansei Gakuin University. 


\section{References}

[1] D. S. Kasir. The Algebra of Omar Khayyam (English translation of Khayyam's original work Al-Jabr W'al Muqâbalah). Teachers College Press, 1931.

[2] P. L. Wantzel. Recherches sur les moyens de connaître si un problème de géométrie peut se résoudre avec la règle et le compas. Journal de Mathématiques Pures et Appliquées, pages 366-372, 1837.

[3] H. Huzita. Axiomatic Development of Origami Geometry. In Proceedings of the First International Meeting of Origami Science and Technology, pages 143-158, 1989.

[4] R. C. Alperin. A Mathematical Theory of Origami Constructions and Numbers. New York Journal of Mathematics, 6:119-133, 2000.

[5] T. Ida, A. Kasem, F. Ghourabi, and H. Takahashi. Morley's Theorem Revisited: Origami Construction and Automated Proof. Journal of Symbolic Computation, 46(5):571 - 583, 2011.

[6] J. Justin. Résolution par le pliage de l'équation du troisième degré et applications géométriques. In Proceedings of the First International Meeting of Origami Science and Technology, pages 251-261, 1989.

[7] F. Ghourabi, A. Kasem, and C. Kaliszyk. Algebraic Analysis of Huzita's Origami Operations and their Extensions. In Automated Deduction in Geometry, LNAI/LNCS. Springer, 2013. (to appear).

[8] F. Ghourabi, T. Ida, H. Takahashi, M. Marin, and A. Kasem. Logical and Algebraic View of Huzita's Origami Axioms with Applications to Computational Origami. In Proceedings of the 22nd ACM Symposium on Applied Computing (SAC'07), pages 767-772, Seoul, Korea, 2007.

[9] R. C. Alperin and R. J. Lang. One-, Two, and Multi-fold Origami Axioms. In Origami ${ }^{4}$, Proceedings of the Fourth International Meeting of Origami Science, Mathematics, and Education (4OSME), pages 371-393, 2009.

[10] F. Ghourabi, T. Ida, and H. Takahashi. Computational Origami of Angle Quintisection. In Proceedings of the Workshop in Symbolic Computation in Software Science (SCSS2008), RISC Technical Report Series, 08-08, pages 57-68, 2008.

[11] H. M. Cundy and A. P. Rollett. Mathematical Models. Oxford University Press, 1961.

[12] J. K. Brunton. Polygonal Knots. The Mathematical Gazette, 45(354):299-301, 1961.

[13] K. Sakaguchi. On Polygons Made by Knotting Slips of Paper. Technical Report of Research Institute of Education, Nara University of Education, 18:55-58, 1982.

[14] J. Maekawa. Introduction of the study of knot tape. In Origami ${ }^{5}$, Proceedings of the Fourth International Meeting of Origami Science, Mathematics, and Education (5OSME), pages 395403, 2011.

[15] D. A. Cox. Galois Theory. Wiley-Interscience, 2004.

[16] F. Ghourabi, T. Ida, and A. Kasem. Proof Documents for Automated Origami Theorem Proving. In Automated Deduction in Geometry, volume 6877 of LNCS, pages 78-97. Springer, 2011. 